\title{
FATORES DE RISCO PARA ÚLCERA DE PRESSÃO EM PACIENTES ACAMADOS'
}

\author{
RISK FACTORS FOR PRESSURE ULCER IN BEDRIDDEN PATIENTS \\ FACTORES DE RIESGO PARA ÚLCERA DE \\ PRESIÓN EN PACIENTES ENFERMOS
}

\section{Maria do Socorro Moura Lins Silva Telma Ribeiro Garcia ${ }^{3}$}

RESUMO: Estudo exploratório-descritivo, em que se objetivou identificar, na literatura especializada, que condições predisponentes e que fatores de risco estariam relacionados com o desenvolvimento de úlceras de pressão; e verificar com que freqüência essas condições predisponentes e fatores de risco se apresentariam em pacientes acamados, internados em uma instituição prestadora de serviços de saúde, localizada na cidade de João Pessoa-PB. A partir da análise dos resultados obtidos, concluiuse que há a necessidade de construção de um instrumento de medida desse risco que seja sensivel à nossa realidade e que incorpore fatores de risco identificados com uma freqüência significativa neste estudo e que não estão contemplados na maioria das escalas disponiveis na literatura.

PALAVRAS-CHAVE: Úlcera de pressão, Risco para úlcera de pressão

ABSTRACT: Exploratory-descriptive study in which we aimed to identify, in the specialized literature, which predisposing conditions and risks factors would be related to the development of pressure ulcer, and to verify how often these predisposing conditions and risk factors would be present in bedridden patients, hospitalized in an institution that renders health services in João Pessoa- PB. From the analysis of results obtained, we concluded that there is the need to construct an instrument to measure this risk must suit our reality and that incorporates risk factors identified with a significant frequency in this study and are not contemplated in most of the available scales in the literature.

KEYWORDS: Pressure ulcer, Risk for pressure ulcer

RESUMEN: Estudio exploratorio-descriptivo en que se objetivó identificar, en la literatura especializada, que condiciones predisponientes y que factores de riesgo estarian relacionados con el desarrollo de úlceras de presión; y verificar con que freqüencia esas condiciones predisponientes y factores de riesgo se presentarian en pacientes enfermos, ingresados en una instituición prestadora de servicios de salud, ubicada en la ciudad de João Pessoa- PB. A partir del analisis de los resultados obtenidos, se concluyó que hay la necesidad de construcción de un instrumento de medida de ese riesgo que sea sensible a nuestra realidad y que incorpore factores de riesgo identifica dos con una freqüencia significativa en este estudio y que no están contemplados en la mayoría de las escalas disponibles en la literatura.

PALABRAS CLAVE: Úlcera de presión, riesgo para úlcera de presión

\footnotetext{
${ }^{1}$ Resumo de Dissertação apresentada ao Curso de Mestrado em Enfermagem, área de concentração em Enfermagem de Saúde Pública, Centro de Ciências da Saúde, Universidade Federal da Paraiba.

${ }^{2}$ Enfermeira, Mestre em Enfermagem pela UFPB, Professora da Escola de Enfermagem de Nível Médio, Centro de Ciências da Saúde, Universidade Federal da Paraiba.

${ }^{3}$ Enfermeira, Doutora em Enfermagem pela EERP-USP, Professora Adjunto IV, Departamento de Enfermagem de Saúde Públicae Psiquiatria, Centro de Ciências da Saúde, Universidade Federalda Paraiba.
} 


\section{INTRODUÇÃO}

A úlcera de pressão é uma preocupação secular dos profissionais que prestam assistência a pacientes acamados, sendo sua prevenção e tratamento um desafio para a equipe de enfermagem. Dealey (1996) a descreve como sendo "uma lesão localizada na pele provocada pela interrupção do fornecimento de sangue para a área, geralmente provocada porpressão, cisalhamento ou fricção ou uma combinação dos três". Tiago (1996) reforça esta descrição acrescentando que "a pele, o tecido, o músculo e até o osso sofrem um processo destrutivo pela oclusão da circulação sangüínea ocasionada pela pressão demorada".

A úlcera de pressão é considerada uma ferida crônica, por ser de longa duração e reincidência freqüente, e de cicatrização difícil, apesar dos cuidados da equipe de saúde. Embora não ameace diretamente a vida, representa um problema para os indivíduos afetados, uma vez que lhes acarreta considerável desconforto e influência no aumento de dias de permanência no hospital, dificultando seu retorno ao convívio familiar. Também induz à necessidade de tratamentos cirúrgicos e fisioterápicos, além de afetar a auto-imagem e auto-estima dos pacientes, levandoos a evidenciar problemas emocionais, psico-sociais e econômicos.

Durante muito tempo a enfermagem carregou a culpa pela ocorrência das úlceras de pressão, sendo a mesma considerada como resultante de uma assistência de enfermagem precária. Hoje, a equipe de saúde tem consciência da série' de fatores que podem influenciar a resposta tecidual à pressão. A busca desses fatores deve ser uma preocupação de toda a equipe, tendo-se como meta a eliminação ou a redução do risco do paciente para desenvolver a úlcera, através da instituição de estratégias de prevenção apropriadas.

Diante desta constatação, este estudo teve como objetivos específicos:

-identificar, na literatura especializada, que condições predisponentes e que fatores de risco estariam relacionados com o desenvolvimento de úlceras de pressão; e

- verificar com que freqüência essas condições predisponentes e fatores de risco se apresentariam em pacientes acamados, internados em uma instituição prestadora de serviços de saúde.

\section{MATERIAL E MÉTODO}

O estudo proposto foi do tipo exploratório-descritivo. Foi desenvolvido na Enfermaria de Clínica Médica, alas A e B, do Hospital Universitário Lauro Wanderley (HUWL), situado no Campus I da Universidade Federal da Paraíba (UFPB), na cidade de João Pessoa - PB, após permissão da Comissão de Ética e de Pesquisa do referido hospital.

A população de estudo foi constituída por todos os pacientes acamados, internados na Enfermaria de Clínica Médica do HULW/UFPB, no período de novembro de 1997 a fevereiro de 1998. A amostra, do tipo intencional, foi constituída por 52 pacientes e, no plano de seleção de amostra, observaram-se os seguintes critérios de 
inclusão: ser o paciente acamado; estar com a mobilidade física prejudicada (condição esta imposta por prescrição médica, por uso de aparelho ortopédico ou por patologias); ser indicado pela enfermeira do plantão como sendo paciente de risco para úlcera de pressão e ter mais de 15 anos de idade.

Em relação aos aspectos éticos da pesquisa envolvendo seres humanos, foram adotadas as recomendações do Ministério da Saúde, na Resolução № 196, de 10 de outubro de 1996, em especial o inciso III, alínea $\boldsymbol{g}$ - "contar com o consentimento livre e esclarecido do sujeito da pesquisa e/ou do seu representante legal". (BRASIL, 1996).

Para a coleta de dados, foi construído um instrumento contendo duas partes. $\mathrm{Na}$ primeira, incluíram-se informações demográficas dos pacientes, tais como idade, sexo, hipóteses diagnósticas à internação, data e tempo de internação. $\mathrm{Na}$ segunda, uma listagem das condições predisponentes (ou fatores associados) e dos fatores de risco intrínsecos e extrínsecos relacionados com a formação de úlcera de pressão, identificados durante a revisão da literatura que foi empreendida.

A coleta de dados foi realizada por duas pesquisadoras que, seqüencialmente e independentemente, pesquisavam os prontuários, examinavam e observavam os pacientes selecionados. Não houve comunicação entre as duas durante a observação e o exame físico. Os dados obtidos foram tabulados e submetidos à análise estatística através do cálculo do percentual médio de ocorrência das condições predisponentes e dos fatores de risco intrínsecos e extrínsecos, e do índice Kappa (k) e seu intervalo de confiança (IC) para determinar a confiabilidade dos dados obtidos pelas duas pesquisadoras.

\section{RESULTADOS}

Foram estudados 52 pacientes acamados, de ambos os sexos, com idade entre 18 e 92 anos, e com tempo de internação variando entre 01 e 80 dias. $\mathrm{Na}$ distribuição da amostra por sexo, houve uma predominância de pacientes do sexo feminino (63,5\%). Com relação à composição por faixa etária, prevaleceram os pacientes mais velhos - a freqüência percentual acumulada das faixas etárias de 61 a 80 e de 81 anos ou mais foi igual a 59,6\%; a média de idade foi de 62 anos e a mediana de 66 anos.

Na distribuição por dias de internação, observou-se haver predominância de pacientes com 10 a 30 dias de internação $(42,3 \%)$ e com menos de 10 dias de internação (40,4\%). Na amostra total, a média de tempo de internação foi de 18,4 e a mediana de 11 dias. Com relação às hipóteses diagnósticas anotadas nos prontuários dos pacientes por ocasião de admissão, destacaram-se o acidente vascular cerebral, o diabetes mellitus, as neoplasias e a hipertensão arteria. 


\section{1 CONDIÇÕES PREDISPONENTES PARA ÚLCERA DE PRESSÃO}

TABELA 1 - Índice de concordância entre observadores e distribuição do percentual médio de condições predisponentes para úlcera de pressão em pacientes acamados, internados na Enfermaria de Clínica Médica do HULW/UFPB - João Pessoa, nov./97 a fev./98.

\begin{tabular}{|c|c|c|c|c|c|c|}
\hline \multirow{2}{*}{ Condições Predisponentes Para Úlcera De Pressão } & \multicolumn{2}{|c|}{$f$} & \multirow[t]{2}{*}{ k } & \multirow[t]{2}{*}{ IC } & \multirow{2}{*}{$\begin{array}{c}\% \\
(n=52)\end{array}$} & \multirow[t]{2}{*}{ IC } \\
\hline & $\mathrm{O}_{1}$ & $\mathrm{O}_{2}$ & & & & \\
\hline Alterações Metabólicas & 14 & 14 & 1,00 & - & 26,9 & 6,15 \\
\hline Alterações Cárdio-respiratórias & 07 & 07 & 1,00 & - & 13,5 & 4,74 \\
\hline Alterações Neurológicas & 26 & 26 & 1,00 & - & 50,0 & 6,93 \\
\hline Alterações Crônico-degenerativas & 21 & 21 & 1,00 & - & 40,3 & 6,80 \\
\hline Alterações Nutricionais & 43 & 41 & 0,87 & 0,047 & 80,8 & 5,46 \\
\hline Alterações Circulatórias & 30 & 29 & 0,92 & 0,038 & 56,7 & 6,87 \\
\hline Alterações Hematológicas & 44 & 44 & 1,00 & - & 84,6 & 5,01 \\
\hline Alterações Psicogênicas & 29 & 32 & 0,88 & 0,045 & 58,7 & 6,83 \\
\hline Uso de Medicamentos Depressores do SNC & 34 & 34 & 1,00 & - & 65,4 & 6,60 \\
\hline
\end{tabular}

Observa-se na Tabela 1 que $84,6 \%$ dos pacientes apresentavam alterações hematológicas. Entre essas alterações destacaram-se a anemia, evidenciada em $61,5 \%(I C=6,75)$, a leucocitose em $55,8 \%(I C=6,89)$ e a leucopenia em $9,6 \%$ $(I C=4,09)$. A anemia resulta de uma redução de células vermelhas do sangue (eritrócitos), diminuindo assim, a capacidade de transporte do oxigênio. O paciente com anemia pode apresentar palidez, cicatrização demorada das feridas, fadiga, fraqueza, anorexia, perda de peso, entre outras manifestações, o que pode facilitar o desenvolvimento de úlceras de pressão. (Smeltzer; Bare, 1994). Quanto às alterações nas células brancas, Miller (1991) afirma que estas células "desempenham papel essencial no mecanismo de defesa do organismo contra agressões infecciosas ou de outra natureza". Representam, portanto, um fator importante para a defesa contra as agressões à pele, de que é exemplo a úlcera de pressão.

As alterações nutricionais estavam presentes em $80,8 \%$ dos pacientes. Elas podem ser causadas por "uma dieta insuficiente ou ingestão excessiva de alimentos, por uma dieta desequilibrada, pelo uso aumentado de um nutriente específico ou uma falha do organismo na sua utilização". (Hood; Dincher, 1995). Os nutrientes são essenciais para o crescimento, funcionamento normal das células do organismo e regeneração tissular. Quando ocorrem alterações, como a carência de alguns nutrientes essenciais, o organismo pode tornar-se incapaz de crescer, manter-se ou regenerar-se. A deficiência dos nutrientes, a exemplo das vitaminas e proteínas, deixa os tecidos susceptíveis à integridade da pele prejudicada, quando expostos à pressão (Atkinson; Murray, 1989, Bryant et al., 1992).

Entre as alterações nutricionais, observou-se que 51,0\% (IC =6,93) dos pacientes apresentavam emagrecimento, e 24,0\% (IC =5,92) desnutrição. O 
emagrecimento é uma condição na qual o peso está abaixo dos valores mínimos normais e a desnutrição se caracteriza pela deficiência protéico-calórica. Dentre as suas manifestações clínicas, o paciente pode apresentar a redução de massa muscular e tecido subcutâneo, astenia, irritabilidade, edema generalizado, lesões cutâneas, pele seca, cabelos quebradiços. Os pacientes desnutridos são vulneráveis à formação de úlcera de pressão e, quando estas se instalam, resistem à cicatrização. (Beyers; Duda, 1989, Porto, 1996).

Por sua vez, 5,8\% (IC = 3,24) dos pacientes apresentavam obesidade, caracterizada pelo peso corpóreo de $20 \%$ ou mais acima do peso ideal e o panículo adiposo ultrapassando os limites da normalidade. O paciente obeso, quando acamado, é mais difícil de mobilizar-se ou ser mobilizado, sendo, quase sempre, arrastado na cama durante a mobilização, o que pode levar à lesão dos tecidos. (Atkinson; Murray, 1989, Dealey, 1996, Porto, 1996).

Em relação ao uso de medicamentos depressores do sistema nervoso central, observou-se que $65,4 \%$ dos pacientes faziam uso deste tipo de medicação, dos quais $46,2 \%(I C=6,91)$ faziam uso de analgésicos, utilizados para proporcionar alívio da dor e promover relaxamento, enquanto $19,2 \%(I C=5,46)$ faziam uso de tranqüilizantes, que reduzem a angústia e as preocupações, sendo em geral prescritos para pacientes agitados, ansiosos e hiperativos. Esses dois grupos de medicamentos induzem ao sono e, conseqüentemente, os pacientes podem permanecer por mais tempo numa mesma posição, aumentando o risco para a formação de úlcera de pressão. (Asperheim, 1994).

Como foi observado na Tabela 1, 58,7\% dos pacientes apresentavamalterações psicogênicas, entre as quais destacou-se a desorientação, identificada em 46,2\% (IC =6,91) deles. Segundo a literatura revisada, a desorientação se caracteriza pela perda da noção de tempo, espaço e, às vezes, também da noção do esquema corporal (Porto, 1996). Ela pode predispor o paciente ao risco de desenvolver úlcera de pressão, por ele apresentar um nível prejudicado de consciência que o leva a não perceber a necessidade de reposicionar o corpo para aliviar a pressão. Por sua vez, $9,6 \%(I C=4,09)$ dos pacientes, além da desorientação, apresentavam também agitação. O paciente agitado pode repetidamente friccionar e pressionar o corpo contra os lençóis o que irá favorecer a formação de úlcera de pressão.

Uma outra alteração psicogênica presente foi a depressão, evidenciada em $12,5 \%(I C=4,59)$ dos pacientes e que se caracteriza por uma tristeza profunda e sem motivo aparente, acompanhada de inibição ou lentidão dos processos psíquicos, levando o paciente a apresentar pobreza de movimentos, mímica apagada, inapetência, astenia, tristeza, perda de vontade de viver, desinteresse por si e pelo que acontece ao seu redor, entre outras manifestações. (D’Andréa, 1990). O paciente com depressão, em geral, pode permanecer em uma mesma posição por um tempo prolongado, ficando, assim, vulnerável à formação de úlcera de pressão (Hirschfeld; Goodwin, 1992, Porto, 1996).

Observou-se que $56,7 \%$ dos pacientes acamados apresentavam alteração circulatória, evidenciada pelo edema, que ocorre devido ao excesso de líquido acumulado no espaço intersticial dos tecidos que constituem a pele e a tela subcutânea. Ele varia de intensidade, podendo ser determinado através de compressão no local 
(sinal de Cacifo), variando de uma a quatro cruzes. O edema prejudica a circulação, interferindo negativamente no fornecimento de nutrientes para a célula. Os pacientes edemaciados ficam propensos ao desenvolvimento de úlcera de pressão porque "a pele superficial é muito estirada sobre olíquido subjacente acumulado" (Beyers; Duda, 1989, Smeltzer; Bare, 1994, Porto, 1996). Cinqüenta por cento dos pacientes apresentavam alterações neurológicas. O sistema neurológico controla e regula todos os outros sistemas corporais, tendo a função de autoproteção, incluindo a capacidade de pensar, estar consciente, responder apropriadamente ao ambiente, locomover-se; de regulação da temperatura corporal e respostas reflexas protetoras. Quando ocorre a perda desta função de proteção, pode haver sintomas tais como diminuição da capacidade de mover-se e de sentir (Black; Matassarin-Jacobs, 1996). Entre essas possíveis alterações, destacou-se o acidente vascularcerebral (AVC), diagnosticado em 38,5\% $(6,75)$ dos pacientes acamados. No AVC ocorre uma "infartação" (morte) de uma área do cérebro, devido à insuficiência da irrigação sangüínea. O AVC pode ocorrer por oclusão de um dos principais vasos que irrigam o cérebro, por obstrução parcial ou completa de um grande vaso intracraniano, ou por hemorragia cerebral. Como seqüela, podem ocorrer a hemiplegia, alterações cinestésicas, apraxia, incontinência urinária e fecal (ocasionada por desatenção, lapsos de memória, fatores emocionais ou incapacidade de comunicar-se), confusão, esquecimento, sendo todos estes fatores citados contribuintes para a formação de úlcera de pressão. (Black; Matassarin-Jacobs, 1996).

Ainda na Tabela 1, observou-se que $40,3 \%$ dos pacientes apresentavam alterações crônico-degenerativas - 26,9\% (IC = 6,15) apresentavam câncer e 9,6\% (IC = 4,09) artrite. Segundo Black; Matassarin-Jacobs (1996), "as condições crônicas interferem na ingestão, transformação e gasto de energia física para o metabolismo celular, transformação e síntese protéica e funcionamento de sistemas orgânicos". Essas condições podem contribuir para o surgimento da anorexia, fadiga, perda do sono, comprometimento da mobilidade, levando o paciente a um repouso prolongado, o que irá interferir em vários sistemas, inclusive o tegumentar, expondo-o à formação de úlceras de pressão.

Um total de 26,9\% dos pacientes apresentavam alterações metabólicas, evidenciadas pelo diabetes. Segundo Black; Matassarin-Jacobs (1996), as alterações metabólicas referem-se a perturbações das reações bioquímicas que ocorrem dentro do organismo. No diabetes ocorre um desequilíbrio entre o fornecimento e a demanda de insulina, podendo esses pacientes vir a ter complicações vasculares periféricas e diminuição da sensibilidade. Essas complicações são consideradas fatores de risco para a formação de úlceras de pressão.

Por sua vez, 13,5\% dos pacientes apresentavam alterações cárdio-respiratórias. Segundo a literatura, essas alterações levam o organismo a privar-se de oxigênio e de receber uma quantidade suficiente de sangue, deixando os tecidos predispostos à formação de úlceras de pressão. (Hood; Dincher, 1995, Black; Matassarin-Jacobs, 1996). Entre as alterações cárdio-respiratórias, 5,8\% (IC =3,24) tinham o diagnóstico de pneumonia; a doença pulmonar obstrutiva crônica (DPOC) foi observada em 7,7\% 
$(3,70)$ dos pacientes, entre os quais $3,8 \%(I C=2,65)$ tinham o diagnóstico de enfisema pulmonar e 3,8\% (IC =2,65) de asma brônquica.

Em relação às condições predisponentes para úlcera de pressão apresentadas na Tabela 1, verificou-se que, na interpretação do Kappa, a concordância entre observadores foi perfeita em $67 \%$ e ótima em $33 \%$.

\subsection{FATORES DE RISCO INTRÍNSECOS}

TABELA 2 - Índice de concordância entre observadores e distribuição do percentual médio de fatores de risco intrínsecos para úlcera de pressão em pacientes acamados, internados na Enfermaria de Clínica Médica do HULW/UFPB - João Pessoa, nov./97 a fev./98.

\begin{tabular}{|c|c|c|c|c|c|c|}
\hline \multirow{2}{*}{ Fator de Risco } & \multicolumn{2}{|c|}{ f } & \multirow[t]{2}{*}{ k } & \multirow[t]{2}{*}{ IC } & \multirow{2}{*}{$\begin{array}{c}\% \\
(n=52)\end{array}$} & \multirow[t]{2}{*}{ IC } \\
\hline & $\mathrm{O}_{1}$ & $\mathrm{O}_{2}$ & & & & \\
\hline Sensibilidade superficial alterada & 19 & 20 & 0,96 & 0,027 & 37,5 & 6,71 \\
\hline Alteração do turgor e elasticidade da pele & 40 & 41 & 0,82 & 0,053 & 77,9 & 5,75 \\
\hline Alteração na umidade da pele & 41 & 41 & 0,78 & 0,057 & 78,8 & 5,67 \\
\hline Alteração na textura da pele & 24 & 20 & 0,70 & 0,064 & 42,3 & 6,85 \\
\hline Proeminência óssea evidenciada & 28 & 28 & 1,00 & - & 53,8 & 6,91 \\
\hline Idade ${ }^{3} 60$ anos & 32 & 32 & 1,00 & - & 61,5 & 6,75 \\
\hline Alteração na temperatura corporal & 08 & 08 & 1,00 & - & 15,4 & 5,01 \\
\hline Mobilidade física prejudicada total & 19 & 22 & 0,88 & 0,045 & 39,4 & 6,78 \\
\hline Mobilidade física prejudicada parcial & 33 & 30 & 0,88 & 0,045 & 60,6 & 6,78 \\
\hline
\end{tabular}

Conforme observa-se na Tabela 2, 78,8\% dos pacientes apresentavam alteração na umidade da pele, que está relacionada com sua hidratação, referente tanto à água quanto à oleosidade. "A pele em geral é seca, porém não excessivamente, e sua umidade freqüentemente reflete os niveis de umidade e temperatura do ambiente" (Black; Matassarin-Jacobs, 1996).

Entre as alterações na umidade da pele, destacou-se a pele seca, presente em $73,1 \%(I C=6,15)$ dos pacientes, que pode ser também um sinal de desidratação e que se caracteriza pela diminuição de água e eletrólitos totais do organismo. $\mathrm{Na}$ pele seca, com elasticidade diminuída, a tolerância ao calor, à fricção e à pressão é mais baixa, tornando-a susceptível à rotura.

Ainda em relação à alteração na umidade da pele, 5,8\% $(I C=3,24)$ dos pacientes apresentavam a pele sudorenta. Em pacientes acamados, a pele sudorenta também representa risco para a formação de úlceras de pressão, porque a umidade excessiva pode levar à maceração da pele, tornando-a fragilizada.

Do total de pacientes, $77,9 \%$ apresentavam diminuição no turgor e elasticidade da pele, indicando pele desidratada. Ressalte-se que o turgor vai diminuindo com a idade, à medida que a pele perde a elasticidade normal e sua capacidade de permanecer hidratada (Beyers; Duda, 1989). Dessa forma, o percentual encontrado 
justifica-se pela predominância de pacientes idosos na amostra do estudo.

$\mathrm{Na}$ Tabela 2, observou-se que $61,5 \%$ dos pacientes tinham a idade igual ou superior a 60 anos. Os autores estudados, a exemplo de Beyers; Duda (1989), Bryantetal. (1992), Smeltzer; Bare (1994), Declair(1994) e Benbow; Dealey (1996), enfatizam que é comum nas pessoas idosas o aparecimento de alterações neurológicas, sensoriais, metabólicas, endócrinas, circulatórias e diminuição do sistema respiratório. Referem ainda que os idosos tornam-se progressivamente frágeis, apresentando principalmente alterações vasculares e cutâneas, havendo uma diminuição da produção do colágeno e da camada lipídica da pele, uma redução da perfusão dos tecidos e fragilização das estruturas. A maioria apresenta a pele seca, mais fina e com menor elasticidade. Portanto, este grupo de pacientes é considerado de risco para a formação de úlcera de pressão, conforme já foi afirmado anteriormente.

Em relação à mobilidade física, foi observado que $60,6 \%$ dos pacientes apresentavam a mobilidade física prejudicada parcial e 39,4\%, mobilidade física prejudicada total. Segundo Potter; Perry (1996), "a mobilidade é a habilidade que um individuo possui em se movimentar livremente". A habilidade/capacidade para mudar a posição do corpo é essencial para evitar pressão nas áreas da pele que não suportam peso. A imobilidade traz sérias complicações para o indivíduo, tais como alterações cardiovasculares, respiratórias, músculo-esqueléticas e cutâneas. Estas complicações estão associadas ao grau e duração da imobilidade. Os autores estudados são unânimes em citar a mobilidade diminuída ou ausente como um fator de risco importante para o desenvolvimento de úlcera de pressão (Braden; Bergstrom, 1987, Bryant et al., 1992, Smeltzer; Bare, 1994, Dealey, 1996). A mobilidade física prejudicada, seja total ou parcialmente, esteve presente em todos os pacientes pesquisados, o que se justifica pelo fato de a amostragem ter sido do tipo intencional.

Em 53,8\% dos pacientes acamados havia proeminências ósseas evidenciadas. No local das proeminências ósseas o tecido adiposo existe em pequena quantidade, o que o torna menos resistente à pressão prolongada e, portanto, mais susceptível à formação de úlceras de pressão. Como afirmamos anteriormente, 52\% dos pacientes apresentavam emagrecimento, o que justifica o percentual encontrado de pacientes com proeminências ósseas evidenciadas.

Ainda em relação às condições da pele, pôde-se observar na Tabela 2 que $42,3 \%$ dos pacientes apresentavam alterações em sua textura. "A pele deve parecer suave, macia e elástica. Não deve existiráreas de elevações e nem de espessamento ou adelgaçamento (atrofia) incomuns" (Black; Matassarin-Jacobs, 1996). Dos pacientes que apresentavam alteração na textura da pele, 40,4\% $(I C=6,80)$ tinham pele áspera e $1,9 \%(I C=1,90)$ pele lisa ou fina.

Verificou-se que 37,5\% dos pacientes apresentavam sensibilidade superficial alterada. A sensibilidade é a propriedade que possuem certas partes do SNC de receber, transmitir ou perceber impressões. É a capacidade do corpo de responder a um estímulo de qualquer natureza (tátil, térmico ou doloroso) e de responder intencionalmente à mudança de posição ou procurar ajuda para esta mudança. A inabilidade para sentir ou reconhecer a pressão ou desconforto aumenta o risco para 
o desenvolvimento de úlcera de pressão porque "causa uma redução no estímulo para movimentação e é manifestada por uma inatividade física ainda maior". (Bergstrom et al., 1987, Braden; Bergstrom, 1994, Potter; Perry, 1996, Porto, 1996).

Em relação à temperatura corporal, $15,4 \%$ dos pacientes apresentavam alterações, dos quais 9,6\% (IC =4,09) apresentavam hipertermia e 5,8\% (IC = 3,24) hipotermia. Bryant et al. (1992) afirmam que, em diversos estudos, a elevação da temperatura corporal tem sido associada com o risco para o desenvolvimento da úlcera de pressão, mas que o mecanismo da associação entre elevação da temperatura corporal e o desenvolvimento de úlcera ainda não está esclarecido.

Em relação aos fatores de risco intrínsecos relacionados na Tabela 2, verificouse que, na interpretação do Kappa, a concordância entre observadores foi perfeita em $25 \%$, ótima em $50 \%$ e boa em $25 \%$.

\subsection{FATORES DE RISCO EXTRÍNSECOS}

TABELA 3 - Índice de concordância entre observadores e distribuição do percentual médio de fatores de risco extrínsecos para úlcera de pressão em pacientes acamados, internados na Enfermaria de Clínica Médica do HULW/UFPB - João Pessoa, nov./97 a fev./98.

\begin{tabular}{|l|r|r|r|r|r|r|}
\hline \multicolumn{1}{|c|}{ Fatorde Risco } & \multicolumn{2}{|c|}{$\mathbf{f}$} & $\mathbf{k}$ & IC & \% & IC \\
\cline { 2 - 7 } & $\mathbf{O}_{\mathbf{1}}$ & $\mathbf{O}_{\mathbf{2}}$ & & & $\mathbf{( n = 5 2 )}$ & \\
\hline Força de pressão no corpo & 42 & 42 & 1,00 & - & 80,8 & 5,46 \\
\hline Força de cisalhamento & 16 & 15 & 0,95 & 0,031 & 29,8 & 6,34 \\
\hline Restrição do movimento por contensão total ou parcial & 09 & 09 & 1,00 & - & 17,3 & 5,25 \\
\hline Mobilização inadequada & 35 & 35 & 1,00 & - & 67,3 & 6,51 \\
\hline Condições inadequadas do colchão & 27 & 27 & 1,00 & - & 51,9 & 6,93 \\
\hline Condições inadequadas de roupa da cama & 37 & 38 & 0,95 & 0,031 & 72,1 & 6,22 \\
\hline Higiene corporal inadequada & 15 & 15 & 1,00 & - & 28,8 & 6,28 \\
\hline
\end{tabular}

O - Observador $1 \mathrm{O}_{2}$ - Observador 2 - k - Índice Kappa - IC - Intervalo de Confiança de 95\%

Quando alguma parte do corpo é submetida à força de pressão por um período superior a duas horas, os tecidos são privados totalmente do oxigênio e nutrientes necessários à manutenção de sua integridade, causando, em conseqüência, a morte celular e contribuindo, assim, para a formação de úlceras de pressão. Dos pacientes estudados, $80,8 \%$ apresentavam evidências de força de pressão no corpo, como áreas de rubor e/ou marcas dessa força na pele. Vale ressaltar que a compressão dos tecidos é considerada pelos autores pesquisados, a exemplo de Beyers; Duda (1989), Bryant et al., (1992), Needham (1993), Braden; Bergstrom (1987) e Dealey (1996), como o fator de risco básico ou primário na formação de úlceras de pressão.

Em relação às roupas de cama, verificou-se que 72,1\% dos lençóis estavam com dobraduras; com presença de eliminações vesicais ou intestinais; umedecidos por suor ou outros líquidos; ou com presença de restos alimentares e de pele. Conforme recomendam Campedelli; Gaidzinski (1987), os lençóis devem estar 
esticados na cama para evitar as dobras que geram pontos de pressão; devem estar secos, pois a umidade favorece a maceração da pele, enfraquecendo sua barreira natural. Ainda segundo essas autoras, deve-se evitar também a presença de restos alimentares (migalhas) e objetos, como alfinetes, pinças, termômetros, que podem ficar sob o paciente e provocar pressão.

A mudança de decúbito (mobilização) deve ser efetuada de acordo com a necessidade individual de cada paciente. Normalmente, recomenda-se que o paciente seja reposicionado a cada duas horas, com a finalidade: de aliviar e redistribuir a pressão impedindo, assim, a redução do fluxo sangüíneo na pele e tecido subcutâneo; de auxiliar na drenagem de secreções; de estimular a circulação; de exercitar os membros; e de proporcionar conforto. (Campedelli; Gaidzinski, 1987). Segundo Dealey (1996), "para a maioria dos pacientes, a mobilização periódica é tudo o que é necessário para a prevenção das úlceras de pressão". Pôde-se verificar na Tabela 3 que 67,3\% dos pacientes acamados tinham sua mobilização inadequada.

Em relação aos colchões, 51,9\% dos pacientes estavam em leitos com colchões em condições inadequadas. Deve-se ter o cuidado de observar se o tipo de colchão utilizado pelo paciente é indicado ao seu estado de saúde e se está em condições de suportar o peso do paciente, mantendo-o em equilíbrio, sem proporcionar condições que favoreçam o aumento de pressão no corpo. O forro do colchão também deve estar bem esticado pois, quando dobrado, irá produzir pontos de pressão no corpo. Foi observada uma espessura de colchão menor que $13 \mathrm{~cm}$ em $42,3 \%(I C=6,85)$, forro com dobraduras em 11,5\% $(I C=4,42)$ e tipo de colchão inadequado para o paciente em 19,2\% (IC =5,46) dos leitos dos pacientes acamados.

A força de cisalhamento foi um fator de risco presente em $29,8 \%$ dos pacientes. $O$ cisalhamento ocorre em conseqüência de um posicionamento ou mobilização inadequada. Nele, segundo Smeltzer; Bare, (1994) ocorre "uma interrelação das forças gravitacionais (forças que empurram o corpo para baixo)". As camadas de tecido deslizam umas sobre as outras e, como conseqüência, os "vasos esticam e torcem", rompendo a microcirculação da pele e tecido subcutâneo. A fricção e cisalhamento ocorrem sempre em conjunto porque, no momento em que o paciente desliza no leito, o seu corpo é movido contra o lençol, provocando a fricção, que enfraquece a barreira natural da epiderme. (Dealey, 1996).

$\mathrm{Na}$ Tabela 3, observou-se que $28,8 \%$ dos pacientes apresentavam higiene corporal inadequada. A higiene corporal tem como objetivos o conforto do paciente e a estimulação da circulação sangüínea. A manutenção do corpo limpo contribui para a integridade da pele e para que o corpo se defenda da invasão de organismos patógenos. Identificou-se nesses pacientes a presença de suor, fezes, urina, sangue, secreções e/ou restos alimentares aderidos à sua pele.

Por sua vez, $17,3 \%$ dos pacientes encontravam-se imobilizados no leito, dos quais 3,8\% (IC = 2,65) com contensão total e 13,5\% (IC = 4,74) com contensão parcial. Quando o paciente é mantido imóvel, a capacidade de aliviar a pressão através da mudança de decúbito é afetada de maneira importante, deixando-o vulnerável à formação de úlcera de pressão, além das conseqüências para todo o 
organismo, oriundas de uma imobilidade prolongada, como problemas respiratórios, cardiovasculares, alterações metabólicas, entre outras (Potter; Perry, 1996).

Ao analisar os dados da Tabela 3 , verificou-se que a presença de alguns dos fatores de risco extrínsecos para o desenvolvimento de úlceras de pressão observados nos pacientes relacionavam-se com cuidados preventivos, comprovando a necessidade de recursos humanos de enfermagem em quantidade e qualidade adequadas à execução desses cuidados, assim como a importância da sistematização da assistência de enfermagem na prevenção de úlceras de pressão. Outros fatores, por sua vez, diziam respeito ao planejamento hospitalar de recursos materiais que possam garantir o bem-estar dos pacientes, como é o caso da renovação de colchões com limite de tempo de uso ultrapassado e provisão de roupa de cama em quantidade suficiente para sua troca sempre que se fizer necessário.

Em relação aos fatores de risco extrínsecos relacionados na Tabela 3, verificouse que, na interpretação do Kappa, a concordância entre observadores foi perfeita em $71,4 \%$ e ótima em $28,6 \%$.

\section{CONSIDERAÇÕES FINAIS}

Foram estudados 52 pacientes, de ambos os sexos - $63,5 \%$ de mulheres e $36,5 \%$ de homens; com média de 62 e mediana de 66 anos de idade; e com média de 18,4 e mediana de 11 dias de internação. Nesses pacientes, verificou-se que:

- das alterações de saúde que atuam como condições predisponentes para úlcera de pressão, destacaram-se as alterações hematológicas (84,6\%), representadas por anemia $(61,5 \%)$, leucocitose $(55,8 \%)$ e leucopenia $(9,6 \%)$; as alterações nutricionais $(80,8 \%)$, representadas por emagrecimento $(51,0 \%)$, desnutrição $(24,0 \%)$ e obesidade $(5,8 \%)$; e o uso de medicamentos depressores do SNC $(65,4 \%)$, em especial os analgésicos $(46,2 \%)$ e os tranqüilizantes $(19,2 \%)$;

- entre os fatores de risco intrínsecos, predominaram a alteração na umidade da pele (78,8\%); a alteração no turgore na elasticidade da pele $(77,9 \%)$; a idade $\geqslant 60$ anos $(61,5 \%)$; e a mobilidade física parcialmente prejudicada (60,6\%);

- entre os fatores de risco extrínsecos, sobressaíram-se a força de pressão no corpo $(80,8 \%)$; as condições inadequadas de roupa da cama (72,1\%); a mobilização inadequada (67,3\%); e as condições inadequadas do colchão $(51,9 \%)$;

- a força de cisalhamento, um fator de risco extrínseco que, junto com a força de pressão no corpo, é referida como básica para o desenvolvimento da úlcera de pressão, foi observada em $29,8 \%$ ( $\pm 6,34)$, fato que não diminui sua importância e necessidade de inclusão em instrumentos de mensuração do risco para úlcera de pressão.

Vale ressaltar que os fatores de risco intrínsecos que foram observados com percentual de ocorrência mais alto estão relacionados com a característica etária predominante nos pacientes investigados, ou seja, idade $>60$ anos, reconhecidamente uma parcela da população mais vulnerável ao desenvolvimento de úlceras de pressão, haja vista as alterações orgânicas decorrentes do envelhecimento.

Por sua vez, alguns dos fatores de risco extrínsecos identificados como 
estando presentes na situação de paciente acamado, a exemplo de imobilidade, força de pressão no corpo e força de cisalhamento, são prevenidos com a estratégia de mobilização a intervalos regulares - uma das tarefas atribuídas à equipe de enfermagem; ou com o uso de colchões especiais (de espuma, ar estático, ar que se alterna, gel, ou colchão d'água), de materiais de posicionamento (travesseiros ou almofadas de espuma) e de auxílio para mobilização (trapézio).

Autores como Meneghin; Lourenço (1998) afirmam que "úlceras de pressão são sinônimos de deficiência na qualidade da assistência de enfermagem prestada." A nosso ver, afirmativas como essa penalizam excessivamente a Enfermagem por não levar em consideração aspectos administrativos e/ou organizacionais dos serviços de saúde que, certamente, estão envolvidos na questão da qualidade da assistência. Inclui-se aqui a adequação qualitativa e quantitativa de recursos humanos e materiais para a prestação de um cuidado de enfermagem livre de riscos para o paciente. É o caso identificado nesse estudo de condições inadequadas de colchão e de roupa de cama, cuja solução foge à alçada da enfermagem. Para a maioria de nossas instituições, esses seriam, lamentavelmente, fatores a serem incluídos em um instrumento de avaliação de risco para desenvolvimento de úlcera de pressão.

Evidentemente, cabe aos profissionais de enfermagem, que são aqueles que permanecem a maior parte do tempo ao lado dos pacientes, entre outras responsabilidades, a manutenção da integridade da pele, sendo necessário para isto, que se estabeleçam condutas que garantam a ausência ou que minimizem a ação dos fatores que contribuem para a alteração dessa integridade. Acreditamos que os aspectos abordados neste estudo são fundamentais para se entender a importância do conhecimento das estruturas e funções da pele pelas enfermeiras, para que possam atuar na prevenção de danos à sua integridade.

Para garantir uma assistência de enfermagem qualificada a pacientes acamados, tendo como meta a prevenção da ocorrência de úlceras de pressão, tem sido recomendada a utilização de escalas para avaliação do risco a que essa clientela está submetida. A partir da revisão da literatura que foi empreendida e dos resultados que foram obtidos neste estudo, temos reforçada a idéia de que há a necessidade de construção de um instrumento de medida desse risco que seja sensível à nossa realidade e que incorpore fatores de risco identificados com uma freqüência significativa, alguns dos quais não-estão contemplados nas escalas divulgadas na literatura.

\section{REFERÊNCIAS BIBLIOGRÁFICAS}

1. ASPERHEIM, M. K. Farmacologia para enfermagem. 7.ed. Rio de Janeiro: Guanabara Koogan, 1994.

2. ATKINSON, L. D.; MURRAY, M. E. Fundamentos de enfermagem: introdução ao processo de enfermagem. Rio de Janeiro: Guanabara Koogan, 1989. 
3. BENBOW, M.; DEALEY, C. Factores extrínsecos que afectam os cuidados com feridas crônicas. Nursing, v.8, n.95, p.30-34, jan. 1996.

4. BERGSTROM, N. et al. The Braden scale for predicting pressure sore risk. Nursing Research, v.36, n. 4, p.205-210, July/Aug. 1987.

5. BEYERS, M.;DUDA, S. Distúrbios comuns da pele. In: Enfermagem médico-cirúrgica: tratado de prática clínica. 2.ed. Rio de Janeiro: Guanabara Koogan, 1989. Cap.46, p.1069-1073.

6. BLACK, J. M.; MATASSARIN-JACOBS, E. Luckmann \& Sorensen enfermagem médico-cirúrgica: uma abordagem psicofisiológica. 4.ed. Rio de Janeiro: Guanabara Koogan, 1996. 2 v.

7. BRADEN, B.;BERGSTROM, N. A conceptual schema for the study of the etiology of pressure sore. Rehabilitation Nursing, v.12, n.1, p.8-12, Jan./Feb. 1987.

8. Predictive validity of the Braden scale for pressure sore risk in a nursing home population. Research in Nursing \& Health, n.17, p.459-470, 1994.

9. BRASIL, Conselho Nacional de Saúde. Resolução n. ำ196, de 10 de outubro de 1996 - Dispõe sobre a realização de pesquisas com seres humanos. Brasília, 1996.

10. BRYANT, R. A. Acute chronic wounds: nursing management. St. Louis: Mosby - Year Book, 1992.

11. BRYANT, R. A. et al. Pressure ulcer. In: Acute chronic wounds: nursing management. St. Louis: Mosby - Year Book, 1992. Chapter 5, p. 105-159

12. CAMPEDELLI, M. C.;GAIDZINSKI, R. R. Escara: problema na hospitalização. São Paulo: Ática. 1987.

13. D'ANDREA, F. F. Transtornos psiquiátricos do adulto. 4.ed. Rio de Janeiro: Bertrand Brasil, 1990.

14. DEALEY, C. Cuidando de feridas: um guia para as enfermeiras. São Paulo: Atheneu, 1996.

15. DECLAIR, V. Aplicação do triglicerídios de cadeia média (TCM) na prevenção de úlceras de decúbito. Rev. Bras. Enferm., Brasília, v.47, n.1, p.27-30, 1994.

16. Úlceras de pressão. São Paulo: SANIPLAN, 1997.

17. HIRSCHFELD, R. M. A.;GOODWIN, F. K. Transtornos do humor. In: TALBOTT et al. Tratado de psiquiatria. Porto Alegre: Artes Médicas, 1992. Cap.13, p.301-328. 
18 HOOD, G. H.;DINCHER, J. R. Fundamentos da prática da enfermagem: atendimento completo ao paciente. 8.ed. Porto Alegre: Artes Médicas, 1995.

19. MENEGHIN, P.;LOURENÇO, T. N. A utilização da escala de Braden como instrumento para avaliar o risco de desenvolvimento de úlceras de pressão em pacientes de um serviço de emergência. Nursing, v.1, n.4, p.13-19, set. 1998.

20. MILLER, O. et. al. Laboratório para o clínico. 7.ed. Rio de Janeiro: Atheneu, 1991.

21. NEEDHAM, J. F. Maintaining skin integrity. In: Gerontological nursing: a restorative approach. New York: Delmar Publishers, 1993. Cap. 4, p.103-120.

22. PORTO, C. C. Exame clínico. 3.ed. Rio de Janeiro: Guanabara Koogan, 1996.

23. POTTER, P. A.;PERRY, A. G. Grande tratado de enfermagem prática. São Paulo: Ed. Santos, 1996.

24. SMELTZER, S. C.: BARE, B. G. Brunner \& Suddarth. Tratado de enfermagem médico - cirúrgica. 7.ed. Rio de Janeiro: Guanabara Koogan, 1994.

25 TIAGO, F. Feridas : etiologia e tratamento. 3.ed. Ribeirão Preto: FAEPA, 1996. 